\title{
Blood pressure response to renal denervation is correlated with baseline blood pressure variability: a patient-level meta-analysis
}

\author{
Alexandre Persu ${ }^{a, b, *}$, Daniel Gordin ${ }^{c, d, *}$ Lotte Jacobs $^{e}$, Lutgarde Thijs $^{e}$, Michiel L. Bots ${ }^{f}$, \\ Wilko Spiering ${ }^{g}$, Atena Miroslawska ${ }^{\text {h,i }}$, Jonas Spaak ${ }^{j}$, Ján Rosak, , Mark R. de Jong ${ }^{m}$, Elena Berra ${ }^{a, n}$, \\ Fadl Elmula M. Fadl Elmula ${ }^{\circ}$, Grégoire Wuerzner ${ }^{p}$, Alison H.M. Taylor ${ }^{q}$, Agnieszka Olszanecka ${ }^{r}$, \\ Danuta Czarneckar ${ }^{r}$, Patrick B. Mark ${ }^{q}$, Michel Burnier ${ }^{p}$, Jean Renkin ${ }^{a, b}$, Sverre E. Kjeldsen ${ }^{\circ}$, \\ Jiří Widimskýk ${ }^{k}$, Arif Elvan ${ }^{m}$, Thomas Kahan ${ }^{j}$, Terje K. Steigen ${ }^{j}$, Peter J. Blankestijn ${ }^{\text {s }}$, Ilkka \\ Tikkanen $^{c, t}$, and Jan A. Staessen ${ }^{e, u}$, on behalf of the European Network COordinating research on \\ Renal Denervation (ENCOReD)
}

Background: Sympathetic tone is one of the main determinants of blood pressure (BP) variability and treatment-resistant hypertension. The aim of our study was to assess changes in BP variability after renal denervation (RDN). In addition, on an exploratory basis, we investigated whether baseline BP variability predicted the BP changes after RDN.

Methods: We analyzed 24-h BP recordings obtained at baseline and 6 months after RDN in 167 treatmentresistant hypertension patients (40\% women; age, 56.7 years; mean 24-h BP, 152/90 $\mathrm{mmHg}$ ) recruited at 11 expert centers. BP variability was assessed by weighted SD [SD over time weighted for the time interval between consecutive readings (SDiw)], average real variability (ARV), coefficient of variation, and variability independent of the mean (VIM).

Results: Mean office and 24-h BP fell by 15.4/6.6 and 5.5/ $3.7 \mathrm{mmHg}$, respectively $(P<0.001)$. In multivariableadjusted analyses, systolic/diastolic SDiw and VIM for 24-h SBP/DBP decreased by $1.18 / 0.63 \mathrm{mmHg}(P \leq 0.01)$ and $0.86 / 0.42 \mathrm{mmHg}(P \leq 0.05)$, respectively, whereas no significant changes in ARV or coefficient of variation occurred. Furthermore, baseline SDiw $(P=0.0006)$, ARV $(P=0.01)$, and VIM $(P=0.04)$ predicted the decrease in 24-h DBP but not 24-h SBP after RDN.

Conclusion: RDN was associated with a decrease in BP variability independent of the BP level, suggesting that responders may derive benefits from the reduction in $\mathrm{BP}$ variability as well. Furthermore, baseline DBP variability estimates significantly correlated with mean DBP decrease after RDN. If confirmed in younger patients with less arterial damage, in the absence of the confounding effect of drugs and drug adherence, baseline BP variability may prove a good predictor of BP response to RDN.

Keywords: ambulatory blood pressure measurement, blood pressure variability, renal denervation, resistant hypertension

\begin{abstract}
Abbreviations: ARV, average real variability; BP, blood pressure; CKD-EPI, Chronic Kidney Disease Epidemiology Collaboration; CV, coefficient of variation; eGFR, estimated glomerular filtration rate; ENCOReD, European Network COordinating research on Renal Denervation; MSNA, muscle sympathetic nerve activity; RDN, renal denervation; $\mathrm{rHT}$, treatment-resistant hypertension; SDw, weighted SD; VIM, variability independent of the mean
\end{abstract}

Journal of Hypertension 2017, 35:000-000

apole of Cardiovascular Research, Institut de Recherche Expérimentale et Clinique, Université Catholique de Louvain, ${ }^{b}$ Cliniques Universitaires Saint-Luc, Université Catholique de Louvain, Brussels, Belgium, 'Abdominal Center Nephrology, University of Helsinki and Helsinki University Central Hospital, dFolkhälsan Institute of Genetics, Folkhälsan Research Center, Helsinki, Finland, 'Studies Coordinating Centre, Research Unit Hypertension and Cardiovascular Epidemiology, KU Leuven Department of Cardiovascular Sciences, University of Leuven, Leuven, Belgium, fJulius Center for Health Sciences and Primary Care, University Medical Center Utrecht, '9Department of Vascular Medicine, University Medical Center Utrecht, Utrecht, The Netherlands, ${ }^{h}$ Department of Cardiology, University Hospital of North Norway, Tromsø, 'Cardiovascular Diseases Research Group, UiT The Arctic University of Norway, Norway, 'Karolinskae Institute, Department of Clinical Sciences, Danderyd Hospital, Division of Cardiovascular Medicine, Stockholm, Sweden, ${ }^{\mathrm{k}}$ Third Department of Internal Medicine, General University Hospital and First Faculty of Medicine, Charles University, Prague, 'Cardiocentre University Hospital Královské Vinohrady and Third Faculty of Medicine, Charles University, Prague, Czech Republic, ${ }^{\mathrm{m}}$ Department of Cardiology, Isala Klinieken, Zwolle, The Netherlands, "Department of Medical Sciences, Internal Medicine and Hypertension Division, AOU Città della Salute e della Scienza, Turin, Italy, ${ }^{\circ}$ Department of Cardiology, Ullevål University Hospital, University of Oslo, Oslo, Norway, PService of Nephrology, Lausanne University Hospital, Lausanne, Switzerland, "BHF Glasgow Cardiovascular Research Centre, University of Glasgow, Glasgow, UK, '1st Department of Cardiology, Interventional Electrocardiology and Hypertension, Jagiellonian University Medical College, Krakow, Poland, 'sDepartment of Nephrology, University Medical Center Utrecht, Utrecht, The Netherlands, 'tMinerva Institute for Medical Research, Helsinki, Finland and uR\&D Group VitaK, Maastricht University, Maastricht, The Netherlands

Correspondence to Jan A. Staessen, MD, PhD, Studies Coordinating Centre, Laboratory of Hypertension, University of Leuven, Campus Sint Rafaël, Kapucijnenvoer 35, Block D, Box 7001, BE-3000 Leuven, Belgium. E-mail: jan.staessen@med.kuleuven.be, jan.staessen@maastrichtuniversity.nl

*Alexandre Persu and Daniel Gordin equal contribution.

Received 19 December 2016 Revised 13 August 2017 Accepted 4 September 2017 J Hypertens 35:000-000 Copyright (C) 2017 Wolters Kluwer Health, Inc. All rights reserved.

DOI:10.1097/HJH.0000000000001582 


\section{INTRODUCTION}

$\mathrm{B}$ lood pressure (BP) variability is the result of complex interactions between extrinsic environmental and behavioral factors and intrinsic cardiovascular regulatory mechanisms, both humoral and neural [1,2]. The influence of these different factors is difficult to disentangle. However, it is generally accepted that central sympathetic drive is one of the main determinants of $\mathrm{BP}$ variability $[1,3]$ and treatment-resistant hypertension [4,5]. In particular, studies implementing microneurographic traffic recordings from peroneal nerves, showed a direct relation between 24h BP variability and muscle sympathetic nerve activity (MSNA) [6]. We hypothesized that an intervention targeting to reduce renal sympathetic nerves activity, such as renal denervation (RDN), might decrease BP variability, which if confirmed in the long-run might decrease cardiovascular risk [7]. However, previous studies testing this hypothesis were usually small, monocentric [2,7,8], and applied indices of $\mathrm{BP}$ variability that were heavily dependent on the $\mathrm{BP}$ level [2,7-9]. These studies did therefore not allow to prove or disprove that RDN influenced BP variability. None of the aforementioned studies tested whether BP baseline variability predicted the $\mathrm{BP}$ response to RDN over and above baseline BP.

Furthermore, in view of the modest BP benefits of RDN performed with the unipolar Symplicity catheter [10,11], the identification of responders to RDN is a central issue in the field $[12,13]$. As patients with higher baseline sympathetic tone may respond better to RDN, the identification of an easy-to-determine, noninvasive index of baseline sympathetic activity, likely to predict the $\mathrm{BP}$ response to RDN, is a top research priority $[12,13]$.

In this study, we took advantage of the collaboration within the European Network COordinating research on Renal Denervation (ENCOReD) [14]. We assessed changes in 24-h ambulatory BP variability in response to RDN and investigated whether baseline BP variability predicted the 6-month BP changes induced by RDN. We used ambulatory monitoring as the state-of-the-art technique for the assessment of BP.

\section{METHODS}

\section{Patients}

Following the fifth ENCOReD network meeting, held in Leuven on 31 January 2014, 11 centers volunteered to contribute anonymized data for analysis. The eligibility criteria for RDN at the participating centers complied with the European consensus [15] and have been described previously [14]. Briefly, eligibility criteria for RDN included: optimized treatment with three or more antihypertensive drug classes at the maximal tolerated dose, preferably including a diuretic; a systolic office $\mathrm{BP}$ of at least $140 \mathrm{mmHg}$; a daytime or 24-h SBP of at least 135 or $130 \mathrm{mmHg}$, respectively; an estimated glomerular filtration rate (eGFR) of $30 \mathrm{ml} / \mathrm{min}$ per $1.73 \mathrm{~m}^{2}$ or higher; systematic exclusion of secondary hypertension; suitable anatomy of the renal arteries (diameter $\geq 4 \mathrm{~mm}$, length $\geq 20 \mathrm{~mm}$, absence of stenosis $\geq 50 \%$ or renal artery stent). All patients who underwent at least two 24-h BP measurements of sufficient quality, one at baseline and the second at follow-up and in whom the unedited BP recordings could be made available were eligible for inclusion in this analysis. Ambulatory BP recordings were reviewed for 222 consecutively enrolled patients. Of those, we excluded 52 , due to missing readings during three consecutive hours on ambulatory $\mathrm{BP}$ monitoring, either at baseline or at follow-up. Three additional patients were discarded, because the number of daytime or night-time readings was less than 10 or 5 , respectively. The total number of patients eligible for inclusion in the current analysis was therefore 167. All participating centers received approval from the competent Institutional Review Board. Patients provided written informed consent except in centers in which RDN is part of routine clinical care.

\section{Blood pressure measurement}

In the current RDN studies, office BP was measured either by validated oscillometric devices (10 centers) or auscultation of the Korotkoff sounds (one center). The number of office readings averaged per visit ranged from two to five. All participating centers used validated portable monitors to measure the ambulatory BP according to the guidelines of the European Society of Hypertension [16]. Across centers, the intervals between daytime and night-time readings ranged from 15 to $30 \mathrm{~min}$ and from 30 to $60 \mathrm{~min}$, respectively. The recordings were sparsely edited, removing only readings labeled with an error code or with lower SBP than DBP level. We computed the daytime and night-time BP as the within-individual mean of the readings between 1000 and $2000 \mathrm{~h}$ (daytime) and 0000 to $0600 \mathrm{~h}$ (night-time), respectively, weighted for the interval between readings. These short definitions of daytime and night-time eliminate the transition periods in the morning and the evening during which $\mathrm{BP}$ changes rapidly in most people and result in daytime and night-time BP levels that approximate within $1-2 \mathrm{mmHg}$ to the wakeful and asleep BP recorded by the diary method $[17,18]$.

\section{Blood pressure variability}

We assessed reading-to-reading 24-h BP variability using different estimates, both dependent [weighted SD (SDw), average real variability (ARV)] and independent of the mean [coefficient of variation, variance independent of the mean (VIM)]. Weighted SD [SD over time weighted for the time interval between consecutive readings (SDiw)] is the SD over $24 \mathrm{~h}$ weighted for the time interval between consecutive readings [19,20]. ARV [21] is the average of the absolute differences between consecutive BP measurements. It has the advantage of accounting for the order of the BP measurements. SD weighted according to Bilo et al. [22] (SDrw) is the average of daytime and night-time SD weighted for the duration of the daytime and night-time interval. It allows to get rid of the influence of nocturnal BP fall. However, it remains dependent to some extent of mean BP. Coefficient of variation is SD divided by the mean. Finally, variability independent of the mean (VIM) [23] is calculated as the SD divided by the mean to the power $x$ and multiplied by the population mean to the power $x$. The power $x$ is obtained by fitting a curve through a plot of SD against mean using 
the model $\mathrm{SD}=\mathrm{a} x$ mean $^{x}$, where $x$ was derived by nonlinear regression analysis as implemented in the PROC NLIN procedure of the SAS package (SAS Institute Inc., Cary, North Carolina, USA)

\section{Statistical methods}

We used SAS, version 9.4, for database management and statistical analysis. We applied Student's $t$ tests to compare unadjusted means and to determine the significance of unadjusted within-group BP and BP variability changes (follow-up measurement subtracted from baseline) and the $\chi^{2}$-statistic to compare proportions. To estimate baseline predictors of changes in BP variability, we applied a generalization of the standard linear model, as implemented in the PROC MIXED procedure of the SAS package. In multivariable-adjusted analyses, we considered as covariables: sex, age, BMI, mean arterial pressure, pulse pressure (PP), baseline night-day mean BP ratio, eGFR (estimated according to the Chronic Kidney Disease Epidemiology Collaboration formula) [24], smoking and drinking, and a history of diabetes mellitus or cardiovascular disease. In mixed models, we also adjusted for baseline and we accounted for center as a random effect. Significance was a two-tailed $\alpha$ level of 0.05 or less.

\section{RESULTS}

\section{Baseline characteristics}

Twenty-four-hour ambulatory BP measurements were analyzed in 167 patients (mean age 56.7 years; 40.1\% women; mean baseline office and 24-h ambulatory BP: 172/98 and $152 / 90 \mathrm{mmHg}$, respectively). The median number of ambulatory $\mathrm{BP}$ readings at baseline was 58 (IQR: 45-64; 5 th-95th percentile: 33-78) over $24 \mathrm{~h}, 28$ (IQR: 20-31; 5th-95th percentile: 16-40) during daytime and 12 (IQR: 7-13; 5th-95th percentile: 6-18) during night-time. Corresponding numbers at follow-up were 58 (IQR: 46-65; 5th-95th percentile: 31-77) over $24 \mathrm{~h}, 27$ (IQR: 21-30; 5th-95th percentile: 13-39) during daytime and 12 (IQR: 7-13; 5th-95th percentile: 6-18) during night-time. Table 1 lists the baseline characteristics of the patients across tertiles of 24-h SBP and DBP. The proportion of smokers was higher in the second (20.7\%) and third (20.4\%) tertiles compared with the first $(3.6 \%)$ tertile of SBP $(P=0.016)$. Mean age tended to be higher in the first ( 59.5 years) compared with the second (54.7 years) and third (56.1 years) tertiles $(P=0.053)$. The $24-\mathrm{h}$ ambulatory heart rate (HR) increased significantly across tertiles of 24 -h SBP $(P<0.029)$. Similar differences were observed across tertiles of DBP. SDtw and ARV derived from SBP slightly increased with higher category of 24-h SBP (SDtw: 13.1 \pm 4.6 ; 14.6 \pm 4.3 ; $15.2 \pm 4.4 \mathrm{mmHg} ; \quad P=0.041 ; \quad$ ARV: $12.0 \pm 3.1 ; 13.2 \pm 4.2 ;$ $13.7 \pm 4.2 \mathrm{mmHg} ; \quad P=0.06$ ), whereas no trend was observed for SDiw, coefficient of variation or VIM derived from SBP. SDiw, SDtw, and ARV derived from DBP significantly increased across tertiles of DBP (SDiw: 11.6 \pm 3.6 ; $13.0 \pm 4.3 ; \quad 14.8 \pm 4.3 ; \quad P=0.0004 ; \quad$ SDtw: $8.9 \pm 2.4 ;$ $10.2 \pm 2.8 ; 11.8 \pm 4.2 ; P<0.0001 ;$ ARV : $8.6 \pm 2.6 ; 9.6 \pm 3.1 ;$ $10.8 \pm 4.1 ; P=0.002)$. No trend was observed for coefficient of variation or VIM derived from DBP. Notably, after exclusion of 68 patients with less than 20 daytime and seven night-time BP readings [25], these findings remained virtually unchanged (data not shown).

\section{Renal sympathetic denervation}

Experienced interventional specialists performed all procedures. Symplicity catheters were used in most cases (Symplicity: 72\%, Symplicity Flex: 8\%, Symplicity Spyral: $0.5 \%)$. Other catheters used were 6F short IMA catheters (13\%), Vessix (4\%), St Jude EnlighHTN (2\%), and Covidien OneShot $(0.5 \%)$ catheters.

\section{Experienced changes in blood pressure level after renal denervation}

The SBP/DBP reductions between baseline and follow-up $(6.7 \pm 2.5$ months after RDN) averaged $15.4 / 6.6 \mathrm{mmHg}$ for office BP, and 5.5/3.7 mmHg (Table 2), 6.3/4.1, and 4.5/ $2.9 \mathrm{mmHg}$ for $24-\mathrm{h}$, daytime and night-time ambulatory BP, respectively $(P<0.001$ for all). The 24-h ambulatory $\mathrm{HR}$ decreased from baseline to follow-up with -1.12 [95\% confidence interval (CI): -2.17 to -0.08$] \mathrm{bpm}(P=0.035$ after multivariable adjustment). The number of drug classes decreased from $4.8 \pm 1.5$ at baseline to $4.3 \pm 1.7$ at followup $(P<0.001)$.

\section{Changes in blood pressure variability after renal denervation}

Changes in ARV derived from systolic or diastolic 24-h ambulatory BP did not reach statistical significance ( $-0.20, P=0.49 ;-0.31, P=0.29$, respectively). Similarly, decreases in coefficient of variation were NS $(-0.45$, $P=0.12 ;-0.39, P=0.28$, for $24-\mathrm{h}$ SBP and DBP, respectively). SDiw, SDtw, and VIM derived from 24-h SBP decreased by $-1.29 \mathrm{mmHg}(95 \% \mathrm{CI}:-2.17$ to $-0.42 ; P=$ $0.004),-0.78 \mathrm{mmHg}$ (95\% CI: -1.43 to $-0.12 ; P=0.02$ ), and $-1.11 \mathrm{mmHg}$ (95\% CI: -1.92 to $-0.30 ; P=0.007)$, respectively. Decreases in SDiw and VIM derived from SBP $(-1.18 \mathrm{mmHg}, 95 \% \mathrm{CI}:-1.84$ to $-0.51 ; P=0.0006$ and $-0.86 \mathrm{mmHg}, 95 \% \mathrm{CI}:-1.45$ to $-0.27 ; P=0.005$, respectively) remained significant in multivariable-adjusted analyses and were paralleled by similar changes for 24-h DBP $(-0.63 \mathrm{mmHg}, 95 \% \mathrm{CI}:-1.12$ to $-0.13 ; P=0.014$ and $-0.42 \mathrm{mmHg}$, 95\% CI: -0.86 to $-0.01 ; P=0.054$, respectively) (Table 2).

\section{Relation between baseline BP variability and ambulatory blood pressure changes after renal denervation}

We also tested the relations of $\mathrm{BP}$ variability indices at baseline with changes in ambulatory BP level after RDN, expressed as the difference of 24-ambulatory BP at baseline minus follow-up $(6.7 \pm 2.5$ months after RDN). Although the relation between baseline SDiw derived from 24-ambulatory SBP and change in 24-h systolic ambulatory BP after RDN was borderline significant $(P=0.057)$, no relation was found between other BP variability estimates at baseline and 24-h ambulatory SBP response to $\operatorname{RDN}(P=0.35,0.97$, and 0.25 , for baseline ARV, coefficient of variation and VIM, respectively). In contrast, baseline SDiw $(P=$ 0.0006), ARV $(P=0.01)$, and VIM $(P=0.04)$ - but not coefficient of variation $(P=0.22)$ - derived from $24-\mathrm{h}$ 
TABLE 1. Baseline characteristics of 167 patients across tertiles of 24-h SBP or DBP level

\begin{tabular}{|c|c|c|c|c|c|c|c|c|}
\hline \multirow[b]{2}{*}{ Baseline characteristics } & \multicolumn{4}{|c|}{ 24-h SBP } & \multicolumn{4}{|c|}{ 24-h DBP } \\
\hline & $\leq 142 \mathrm{mmHg}$ & $143-158 \mathrm{mmHg}$ & $\geq 159 \mathrm{mmHg}$ & $P$ value & $\leq \mathbf{8 3} \mathrm{mmHg}$ & $84-95 \mathrm{mmHg}$ & $\geq 96 \mathrm{mmHg}$ & $P$ value \\
\hline Number (\%) with characteristic & 55 & 58 & 54 & & 54 & 56 & 57 & \\
\hline Women & $19(34.6)$ & $20(34.5)$ & $28(51.9)$ & 0.10 & $19(35.2)$ & $22(39.3)$ & $26(45.6)$ & 0.53 \\
\hline Nonwhite ethnicity & $1(1.8)$ & $1(1.7)$ & $4(7.4)$ & 0.19 & $0(0)$ & $2(3.6)$ & $4(7.0)$ & 0.14 \\
\hline Smokers & $2(3.6)$ & $12(20.7)^{* * *}$ & $11(20.4)$ & 0.016 & $3(5.6)$ & $5(8.9)$ & $17(29.8)^{* *}$ & 0.0005 \\
\hline Drinking alcohol & $25(45.5)$ & $22(37.9)$ & $14(25.9)$ & 0.10 & $25(46.3)$ & $20(35.7)$ & $16(28.1)$ & 0.14 \\
\hline Diabetes mellitus & $17(30.9)$ & $11(19.0)$ & $18(33.3)$ & 0.19 & $23(42.6)$ & $15(26.8)$ & $8(14.0)$ & 0.003 \\
\hline \multicolumn{9}{|l|}{ Previous cardiovascular disease } \\
\hline Coronary heart disease & $8(14.6)$ & $11(19.0)$ & $9(16.7)$ & 0.82 & $13(24.1)$ & $8(14.3)$ & $7(12.3)$ & 0.21 \\
\hline Stroke & $2(3.6)$ & $4(6.9)$ & $4(7.4)^{*}$ & 0.66 & $3(5.6)$ & $3(5.4)$ & $4(7.0)$ & 0.92 \\
\hline \multicolumn{9}{|l|}{ Mean (SD) characteristic } \\
\hline Age (years) & $59.5 \pm 11.6$ & $54.7 \pm 9.6^{*}$ & $56.1 \pm 10.8$ & 0.053 & $64.2 \pm 9.6$ & $54.9 \pm 9.5^{* * *}$ & $51.3 \pm 9.2^{*}$ & $<0.0001$ \\
\hline BMI $\left(\mathrm{kg} / \mathrm{m}^{2}\right)$ & $29.6 \pm 4.8$ & $30.0 \pm 5.3$ & $30.2 \pm 5.7$ & 0.83 & $30.4 \pm 5.2$ & $29.8 \pm 5.4$ & $29.7 \pm 5.3$ & 0.73 \\
\hline Serum creatinine $(\mu \mathrm{mol} / \mathrm{l})$ & $88.2 \pm 21.8$ & $85.1 \pm 28.8^{*}$ & $84.3 \pm 25.2$ & 0.70 & $86.2 \pm 19.5$ & $87.3 \pm 29.8$ & $84.2 \pm 25.9$ & 0.81 \\
\hline eGFR $\left(\mathrm{ml} / \mathrm{min}\right.$ per $\left.1.73 \mathrm{~m}^{2}\right)$ & $78.8 \pm 18.0$ & $84.2 \pm 19.7$ & $82.0 \pm 19.5$ & 0.33 & $76.4 \pm 16.9$ & $82.3 \pm 19.6$ & $86.2 \pm 19.7$ & 0.025 \\
\hline $\begin{array}{l}\text { 24-h ambulatory heart rate } \\
\text { Office blood pressure }\end{array}$ & $67.5 \pm 10.9$ & $70.5 \pm 10.2$ & $72.8 \pm 9.7$ & 0.029 & $65.4 \pm 9.4$ & $70.8 \pm 10.5^{* *}$ & $74.3 \pm 9.6$ & $<0.0001$ \\
\hline $\mathrm{SBP}(\mathrm{mmHg})$ & $159.9 \pm 19.1$ & $171.6 \pm 30.1^{*}$ & $184.1 \pm 25.3^{*}$ & $<0.0001$ & $167.9 \pm 26.3$ & $168.1 \pm 24.6$ & $179.1 \pm 28.9^{*}$ & 0.04 \\
\hline $\mathrm{DBP}(\mathrm{mmHg})$ & $91.9 \pm 11.4$ & $98.3 \pm 18.1^{*}$ & $102.4 \pm 18.2$ & 0.004 & $85.5 \pm 11.1$ & $96.6 \pm 12.3^{* * *}$ & $109.9 \pm 15.5^{* * *}$ & $<0.0001$ \\
\hline \multicolumn{9}{|l|}{ Blood pressure variability (SBP) } \\
\hline SDiw $(\mathrm{mmHg})$ & $17.1 \pm 6.2$ & $18.0 \pm 5.0$ & $19.4 \pm 5.7$ & 0.099 & $17.5 \pm 5.9$ & $17.8 \pm 5.7$ & $19.2 \pm 5.4$ & 0.26 \\
\hline SDtw (mmHg) & $13.1 \pm 4.6$ & $14.6 \pm 4.3$ & $15.2 \pm 4.4$ & 0.041 & $13.3 \pm 3.9$ & $14.1 \pm 4.7$ & $15.4 \pm 4.7$ & 0.045 \\
\hline ARV $(\mathrm{mmHg})$ & $12.0 \pm 3.1$ & $13.2 \pm 4.2$ & $13.7 \pm 4.2$ & 0.060 & $12.6 \pm 3.9$ & $12.4 \pm 3.5$ & $13.8 \pm 4.3$ & 0.12 \\
\hline$C V(\%)$ & $12.8 \pm 4.6$ & $11.9 \pm 3.2$ & $11.4 \pm 3.4$ & 0.15 & $12.4 \pm 4.0$ & $12.0 \pm 4.0$ & $11.8 \pm 3.4$ & 0.74 \\
\hline VIM (unit) & $17.8 \pm 6.3$ & $17.5 \pm 4.6$ & $17.6 \pm 5.0$ & 0.97 & $17.4 \pm 5.4$ & $17.4 \pm 5.5$ & $18.1 \pm 5.1$ & 0.75 \\
\hline \multicolumn{9}{|l|}{ Blood pressure variability (DBP) } \\
\hline SDiw $(\mathrm{mmHg})$ & $12.6 \pm 4.4$ & $13.2 \pm 4.0$ & $13.7 \pm 4.3$ & 0.38 & $11.6 \pm 3.6$ & $13.0 \pm 4.3$ & $14.8 \pm 4.3^{*}$ & 0.0004 \\
\hline SDtw $(\mathrm{mmHg})$ & $9.5 \pm 3.1$ & $10.5 \pm 3.9$ & $11.0 \pm 3.2$ & 0.066 & $8.9 \pm 2.4$ & $10.2 \pm 2.8^{*}$ & $11.8 \pm 4.2^{*}$ & $<0.0001$ \\
\hline ARV $(\mathrm{mmHg})$ & $9.1 \pm 3.2$ & $9.6 \pm 3.7$ & $10.3 \pm 3.3$ & 0.066 & $8.6 \pm 2.6$ & $9.6 \pm 3.1$ & $10.8 \pm 4.1$ & 0.002 \\
\hline $\mathrm{CV}(\%)$ & $15.8 \pm 5.5$ & $14.6 \pm 4.1$ & $14.1 \pm 4.4$ & 0.16 & $15.7 \pm 5.2$ & $14.5 \pm 4.7$ & $14.2 \pm 4.3$ & 0.23 \\
\hline VIM (unit) & $13.1 \pm 4.5$ & $12.8 \pm 3.6$ & $12.5 \pm 3.3$ & 0.15 & $12.7 \pm 4.0$ & $12.5 \pm 3.7$ & $13.1 \pm 3.9$ & 0.71 \\
\hline
\end{tabular}

$P$ values denote significance of the differences in prevalence rates or means across tertiles of 24-h SBP or DBP. ARV, average real variability; CV, coefficient of variation; eGFR, glomerular filtration rate estimated from the serum creatinine concentration using Chronic Kidney Disease Epidemiology Collaboration formula; SDiw, SD over time weighted for the

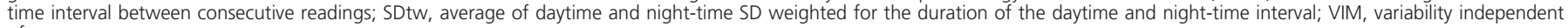
of mean.

Significance of the difference with the adjacent lower tertile:

${ }^{*} P \leq 0.001$

${ }^{* *} P \leq 0.01$

${ }_{* * *} P \leq 0.05$.

DBP were significantly related with changes in 24-h DBP level after RDN. After adjustment for baseline BP, correlations with SDiw $(P=0.028)$ and $\operatorname{VIM}(P=0.030)-$ but not $\operatorname{ARV}(P=0.17)$ - remained statistically significant, while the correlation with coefficient of variation reached statistical significance $(P=0.031)$ (Fig. 1). Finally, we attempted to determine the optimal threshold value of the different BP variability estimates for predicting DBP decrease after RDN. DBP response was defined as a mean 24-h DBP change more than $10 \mathrm{mmHg}$ after RDN. Thresholds were determined by maximizing the Youden index (maximum of sensitivity + specificity -1 ). The optimal thresholds for each baseline variability estimate, the proportion of patients correctly or incorrectly classified as responders or nonresponders, as well as the corresponding sensitivity, specificity, and predictive values are indicated in Table 3.

\section{DISGUSSION}

The two key findings of this new analysis of the ENCOReD database are the following: RDN decreases not only BP level, but BP variability as well; DBP variability at baseline is correlated with 24-h DBP changes in response to RDN.
Several studies [2,7-9] proposed that RDN might decrease BP variability, as captured by the unadjusted [2,7-9] or adjusted [2] SD of mean 24-h ambulatory BP, time-rate of 24-h ambulatory $\mathrm{BP}$ variation (mean of the absolute ratios of the differences between successive BPs and the minutes between them) [8], ARV [2,9] and coefficient of variation of 24-h ambulatory BP [2,9]. However, most of these analyses $[2,7,8]$ were performed in small, single-center cohorts (sample sizes ranging from 11 to 31). The effect of RDN on the indices of BP variability was not always consistent between studies, and some of the indices chosen, such as unadjusted SD of the mean or ARV, are strongly related with mean BP [26]. In all studies $[2,7,9]$ but one [8], RDN was performed using the Symplicity unipolar catheter. Finally, none of these studies assessed VIM, which is considered to be a particularly robust index of $\mathrm{BP}$ variability independent of the mean [23].

In contrast with the publication by Miroslawska et al. [2], performed in a small subset $(n=23)$ of truly adherent patients with resistant hypertension, in the ENCOReD database, RDN was not followed by a significant decrease in ARV or coefficient of variation. Notably however, in the recent study by Ewen et al. [9] including 84 patients, 
TABLE 2. Baseline values and 6-month changes $(\Delta)$ in 24-h blood pressure level and variability indices

\begin{tabular}{|c|c|c|c|c|}
\hline BP level and variability & 24-h Systolic ambulatory BP & P value & 24-h Diastolic ambulatory BP & $P$ value \\
\hline Baseline & $151.6 \pm 16.8$ & & $89.6 \pm 13.4$ & \\
\hline Unadjusted $\Delta$ & $-5.48(-8.12$ to -2.85$)$ & $<0.0001$ & $-3.72(-5.38$ to -2.07$)$ & $<0.0001$ \\
\hline \multicolumn{5}{|l|}{ SDiw (mmHg) } \\
\hline Baseline & $18.2 \pm 5.7$ & & $13.2 \pm 4.3$ & \\
\hline Unadjusted $\Delta$ & $-1.29(-2.17$ to -0.42$)$ & 0.004 & $-0.89(-1.58$ to -0.21$)$ & 0.011 \\
\hline Baseline & $14.3 \pm 4.5$ & & $10.3 \pm 3.5$ & \\
\hline Unadjusted $\Delta$ & $-0.78(-1.43$ to -0.12$)$ & 0.020 & $-0.53(-1.07$ to 0.01$)$ & 0.055 \\
\hline Adjusted $\Delta$ & $-0.43(-0.92$ to 0.06$)$ & 0.087 & $-0.22(-0.64$ to 0.20$)$ & 0.30 \\
\hline \multicolumn{5}{|l|}{ ARV $(\mathrm{mmHg})$} \\
\hline Baseline & $12.9 \pm 4.0$ & & $9.7 \pm 3.4$ & \\
\hline Unadjusted $\Delta$ & $-0.20(-0.78$ to 0.38$)$ & 0.49 & $-0.31(-0.87$ to 0.26$)$ & 0.29 \\
\hline Adjusted $\Delta$ & $-0.38(-0.77$ to 0.01$)$ & 0.059 & $0.01(-0.35$ to 0.38$)$ & 0.94 \\
\hline \multicolumn{5}{|l|}{ VIM (unit) } \\
\hline Baseline & $17.6 \pm 5.3$ & & $12.8 \pm 3.8$ & \\
\hline Unadjusted $\Delta$ & $-1.11(-1.92$ to -0.30$)$ & 0.007 & $-0.77(-1.36$ to -0.18$)$ & 0.011 \\
\hline Adjusted $\Delta$ & $-0.86(-1.45$ to -0.27$)$ & 0.005 & $-0.42(-0.86$ to -0.01$)$ & 0.054 \\
\hline
\end{tabular}

Changes are follow-up - baseline. ARV, average real variability; CV, coefficient of variation; SDiw, SD over time weighted for the time interval between consecutive readings; SDtw, average of daytime and night-time SD weighted for the duration of the daytime and night-time interval; VIM, variability independent of mean.

(a)

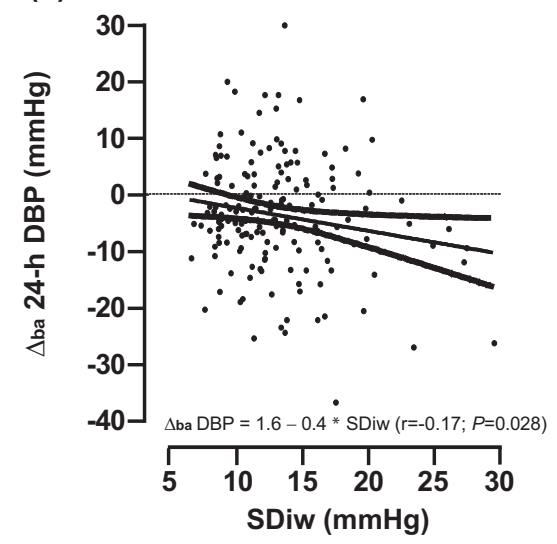

(c)

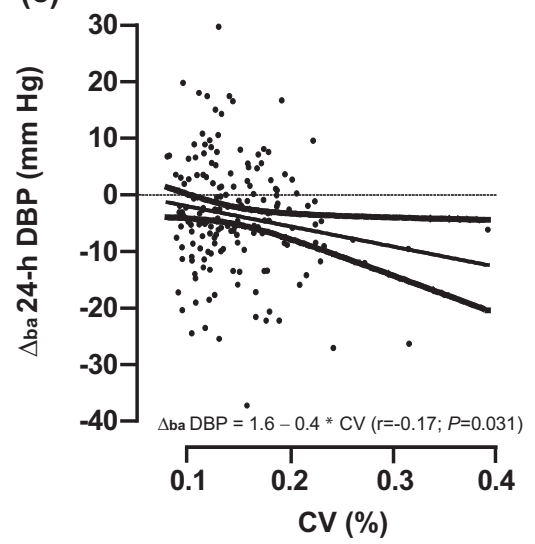

(b)

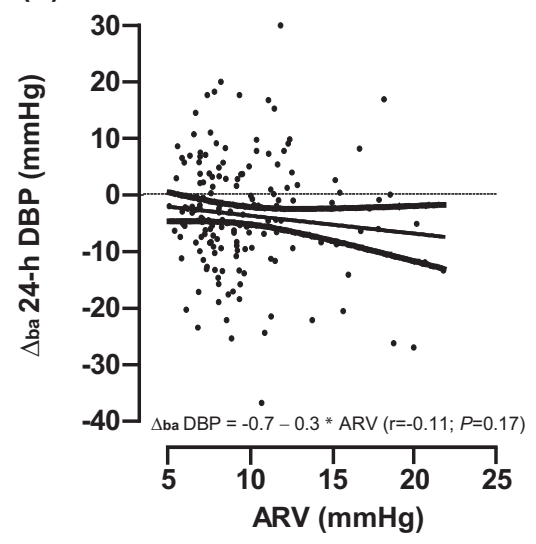

(d)

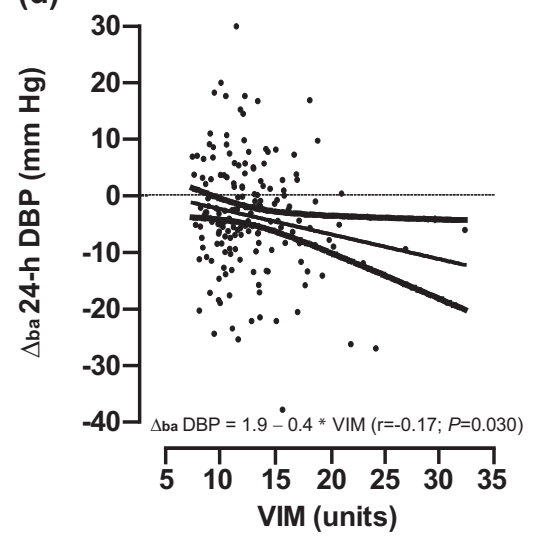

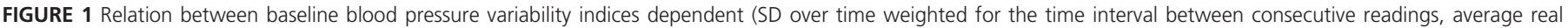

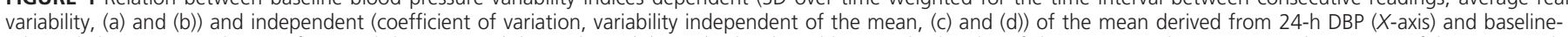

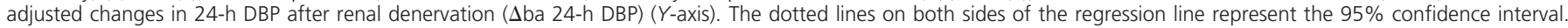
$\mathrm{BP}$, blood pressure. 
TABLE 3. Classification of 24-h DBP level changes after renal denervation $(\Delta)$ by baseline blood pressure variability indices

\begin{tabular}{|c|c|c|c|c|c|c|c|c|}
\hline \multirow[b]{2}{*}{$\begin{array}{l}\text { Blood pressure } \\
\text { variability indices } \\
\text { (threshold }^{\text {a })}\end{array}$} & \multicolumn{2}{|c|}{ Correctly classified } & \multicolumn{2}{|c|}{ Incorrectly classified } & \multicolumn{4}{|c|}{ Classification parameters } \\
\hline & Responder & Nonresponder & Responder & Nonresponder & $\begin{array}{c}\text { Sensitivity } \\
(\%)\end{array}$ & $\begin{array}{c}\text { Specificity } \\
(\%)\end{array}$ & $\begin{array}{l}\text { Positive } \\
\text { predictive } \\
\text { value (\%) }\end{array}$ & $\begin{array}{l}\text { Negative } \\
\text { predictive } \\
\text { value (\%) }\end{array}$ \\
\hline SDiw $(11.8 \mathrm{mmHg})$ & 28 & 66 & 65 & 8 & 77.8 & 50.4 & 30.1 & 89.2 \\
\hline SDtw $(8.7 \mathrm{mmHg})$ & 30 & 59 & 72 & 6 & 83.3 & 45.0 & 29.4 & 90.8 \\
\hline ARV $(7.8 \mathrm{mmHg})$ & 30 & 55 & 76 & 6 & 83.3 & 42.0 & 28.3 & 90.2 \\
\hline CV (15.0\%) & 18 & 83 & 48 & 18 & 50.0 & 63.4 & 27.3 & 82.2 \\
\hline VIM (13.5 units) & 17 & 94 & 37 & 19 & 47.2 & 71.8 & 31.5 & 83.2 \\
\hline
\end{tabular}

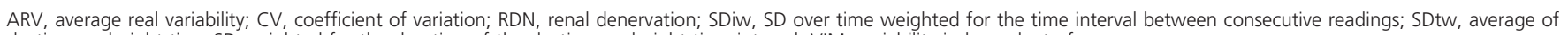
daytime and night-time SD weighted for the duration of the daytime and night-time interval; VIM, variability independent of mean.

${ }^{a}$ Optimal threshold values of the different BP variability indices for predicting DBP changes after RDN were determined by maximizing the Youden index. Responder: mean 24-h DBP decrease more than $10 \mathrm{mmHg}$ after RDN (follow-up - baseline). Nonresponder: mean 24-h DBP decrease $10 \mathrm{mmHg}$ or less.

changes in these measurements were only borderline significant 6 months after RDN $(P=0.054$ and 0.071 , respectively). We nevertheless documented a significant decrease in VIM of 24-h SBP and DBP. The decrease in VIM of 24-h SBP but not DBP remained significant in a fully adjusted model (Table 2). Although most BP variability indices, including coefficient of variation [23] may be influenced by mean BP values, VIM includes an additional coefficient derived from curve fitting which makes it truly independent of the mean $[23,26]$. Along the same lines, SD weighted according to Bilo et al. [22] (SDtw), which allows to get rid of the influence of nocturnal BP fall, also decreased after RDN, though significance was lost after full adjustment. However, it remains dependent to some extent of mean BP. Overall, our results strongly suggest that RDN decreases BP variability over and above its effect on BP level. The lack of decrease in visit-to-visit VIM in the Syst-Eur randomized controlled trial in the placebo and active-treatment arms [27] further supports the hypothesis that decreased BP variability documented after RDN is not entirely explained by reduction of $\mathrm{BP}$ level or regression to the mean, but at least partly reflects the sympatholytic effects of the intervention per se $[1,3]$. In addition to a decrease in BP and BP variability, RDN was also associated with a decrease in HR. These findings are not unexpected in view of the influence of sympathetic system on HR and are in agreement with previous studies [28,29].

Whether decreased BP variability after RDN may contribute to improve cardiovascular prognosis over and above mean BP decrease remains to be demonstrated. First, the possible benefits of RDN in terms of 'hard' cardiovascular endpoints remain unsubstantiated. Second, both changes in BP level [14] and BP variability after RDN using the Symplicity system are modest and highly variable among individual patients. Last but not least, most studies have been performed using BP variability indices that are highly correlated with mean BP [26]. VIM was used in few studies, mostly to assess visit-to-visit BP variability rather than 24-h ambulatory BP variability as in the current study. In the ASCOT study, Rothwell et al. [23] found a strong relation between VIM derived from office BP (visit-to-visit BP variability) - but not ambulatory BP - and cardiovascular and cerebrovascular events. In contrast, in multivariableadjusted analyses, BP variability indices including VIM were not independent predictors of cardiovascular morbidity or mortality, either in the Syst-Eur randomized controlled trial [27] or in a population-based sample representative of the general Flemish population [30].

Another key finding of our study is that baseline diastolic SDiw, coefficient of variation, and VIM correlated with DBP but not SBP response to RDN (Fig. 1). From a pathophysiologic perspective, these results are meaningful. Indeed, the steady component of BP reflected by mean or DBP is a measure of peripheral vascular resistance [31], which in its turn is dependent on sympathetic tone [32] and decreases after renal sympathetic nerve ablation [33]. Several lines of evidence suggest that increased vascular resistance, a hallmark of diastolic hypertension in young patients with sympathetic overactivity [34] is due to narrowing of precapillary arterioles, and that these changes precede BP elevation [35]. In contrast, SBP and PP predominantly reflect the degree of stiffness of conductance vessels [36], increase with the accumulation of aged-related structural damage and are less likely to be influenced by the autonomic system. This may explain the lack of predictive value of baseline SBP variability on SBP changes after RDN, and more generally the modest BP-lowering effects of RDN in patients with isolated systolic hypertension [37]. Along the same lines, it is worth noting that in the recent randomized controlled study DENERVHTA comparing the BP-lowering efficacy of RDN with that of $50 \mathrm{mg}$ of spironolactone in patients with resistant hypertension, the decrease in BP variability was limited to the RDN arm and significant only for DBP not SBP [38]. Notably, the BP reduction associated with RDN precedes and seems to be independent of decrease in sympathetic nerve system activity assessed by MSNA [39]. Hence, the larger BP decrease observed after RDN in patients with a higher baseline BP variability and possibly a higher baseline sympathetic activity - may not be due to a larger decrease in sympathetic nerve system activity. Our results are partly consistent with those obtained by Tsioufis et al. [8] in a cohort of 31 patients denervated using the EnligHTN multielectrode ablation catheter. Nevertheless, comparison is difficult, as the latter used a different BP variability estimate, namely time rate, defined as the first derivative of the BP values against time (mean of the absolute ratios of the differences between successive BPs and the minutes between them).

Our study should be interpreted within the context of its limitations. The most important is the absence of control 
group, which makes it vulnerable to the Hawthorne effect and other patient-related and physician-related bias [40]. However, this limitation is mitigated by the use of $\mathrm{BP}$ variability estimates derived from 24-h ambulatory BP variability, which is blinded by definition, rather than visit-tovisit $\mathrm{BP}$ variability, and inclusion of VIM, which is independent of BP level, and may thus be even less influenced by placebo and white-coat effects. Second, most patients ( $>80 \%)$ were denervated using the first-generation unipolar Symplicity catheter. Hence, our results cannot be extrapolated to more performant, second-generation catheters, which might produce more efficient RDN and therefore larger effects on BP variability. Third, in the absence of procedural endpoint [41], the completeness of RDN could not be assessed. Finally, while the number of prescribed antihypertensive drug classes was documented both at baseline and 6 months after RDN, details on medications and posology were not systematically recorded, and drug adherence was not assessed in most centers [14]. In the absence of the confounding effect of drugs change, the predictive value of baseline $\mathrm{BP}$ variability on $\mathrm{BP}$ response to RDN may be even better. Still, with 167 patients from 11 European centers, our study is the largest performed on BP variability up to now. The mean 24-h BP decrease after RDN $(\sim 5 \mathrm{mmHg})$ is similar to that observed in our initial ENCOReD patient level meta-analysis [14] and in the highly standardized DENERHTN randomized controlled trial [42]. Finally, this study is the first to assess BP variability independent of the mean (VIM), and to look for the predictive value of baseline BP variability on BP outcome after RDN.

In conclusion, RDN using the unipolar Symplicity catheter was associated with a decrease in short-term BP variability at 6 months, over and above a modest decrease in BP level. Whether this would translate into additional benefits in terms of cardiovascular mortality and morbidity remains to be proven. Furthermore, baseline BP variability estimates were related with DBP changes after RDN. This intriguing observation needs confirmation in randomized controlled studies using more efficient and reproducible RDN systems, and/or RDN guided by renal nerve stimulation [41], including younger, ideally untreated patients with milder hypertension, a group considered as particularly suitable for upcoming RDN trials $[11,13]$.

\section{ACKNOWLEDGEMENTS}

The authors are grateful to Dr Qi-Fang Huang (Studies Coordinating Centre, Research Unit Hypertension and Cardiovascular Epidemiology, KU Leuven Department of Cardiovascular Sciences, University of Leuven) for her contribution to the analysis of the data.

Contributing investigators: Brussels, Belgium - Alexandre Persu, Frank Hammer, Sandrine Horman, Joëlle Kefer, Jean-Philippe Lengelé, Jean-Benoit Le Polain de Waroux, Agnès Pasquet, Jean Renkin, Christophe Scavée, Francesca Severino, Christophe Beauloye, and Jean-Louis Vanoverschelde. Glasgow - Collin Berry, Adrian Brady, Christian Delles, Anna Dominiczak, Marie Freel, Alan Jardine, Jon Moss, Scot Muir, Patrick Mark, Sandosh Padmanabhan, and Giles Roditi. Helsinki - Daniel Gordin, Ilkka Tikkanen, and Kimmo Lappalainen. Kraków - Danuta Czarnecka,
Agnieszka Olszanecka, Katarzyna Stolarz Skrzypek, and Piotr Jankowski. Lausanne - Michel Burnier, Grégoire Wuerzner, Salah Qanadli, and Olivier Muller. Leuven, Belgium - Jan A. Staessen, Yu-Mei Gu, Azusa Hara, Lotte Jacobs, Yu Jin, Tatiana Kuznetsova, Lutgarde Thijs, Fang-Fei Wei, Wen-Yi Yang, and Zhen-Yu Zhang. Oslo - Fadl Elmula M Fadl Elmula, Eigil Fossum, Ulla Hjørnholm, Pavel Hoffmann, Aud Høieggen, Vibeke Kjær, Sverre E. Kjeldsen, Anne CK Larstorp, Ingrid Os, Morten Rostrup, and Aud Stenehjem. Prague - Petr Waldauf, Ondrej Petrák, Tomáš Zelinka, Branislav Štrauch, Karol Čurila, Petr Tousek, and Petr Widimský. Stockholm - Malin Hansson, Fadi Jokhaji, Rikard Linder, Thomas Kahan, and Jonas Spaak. Tromsø Atena Miroslawska, Terje K. Steigen, Marit Solbu, and Eystein Skjølsvik. Utrecht - Peter J. Blankestijn, Michiel L. Bots, Wilko Spiering, Michiel Voskuil, and Evert Jan Vonken. Zwolle - de Jong MR, Ahmet Adiyaman, and Arif Elvan.

The European Union (grants IC15-CT98-0329-EPOGH, LSHM-CT-2006-037093-InGenious HyperCare, HEALTH2007-2.1.1-2-HyperGenes, HEALTH-2011.2.4.2-2-EU-MASCARA, HEALTH-F7-305507 HOMAGE and the European Research Council Advanced Researcher Grant-2011294713-EPLORE) and the Ministry of the Flemish Community, Brussels, Belgium (grants G.0881.13, G.088013) supported the Studies Coordinating Centre, Leuven, Belgium. None of the authors received financial support for the current analysis.

\section{Conflicts of interest}

There are no conflicts of interest.

\section{REFERENGES}

1. Parati G, Ochoa JE, Lombardi C, Bilo G. Assessment and management of blood-pressure variability. Nat Rev Cardiol 2013; 10:143-155.

2. Miroslawska A, Solbu M, Skjølsvik E, Toft I, Steigen TK. Renal sympathetic denervation: effect on ambulatory blood pressure and blood pressure variability in patients with treatment-resistant hypertension. The ReShape CV-risk study. J Hum Hypertens 2016; 30:153-157.

3. Parati G, Ochoa JE, Lombardi C, Bilo G. Blood pressure variability: assessment predictive value, and potential as a therapeutic target. Curr Hypertens Rep 2015; 17:537

4. Grassi G, Seravalle G, Brambilla G, Pini C, Alimento M, Facchetti R, et al. Marked sympathetic activation and baroreflex dysfunction in true resistant hypertension. Int J Cardiol 2014; 177:1020-1025.

5. Esler M. Renal denervation for treatment of drug-resistant hypertension. Trends Cardiovasc Med 2015; 25:107-115.

6. Narkiewicz K, Winnicki M, Schroeder K, Phillips BG, Kato M, Cwalina E, Somers VK. Relationship between muscle sympathetic nerve activity and diurnal blood pressure profile. Hypertension 2002; 39:168-172.

7. Zuern CS, Rizas KD, Eick C, Stoleriu C, Bunk L, Barthel P, et al. Effects of renal sympathetic denervation on 24-h blood pressure variability. Front Physiol 2012; 3:1-8.

8. Tsioufis C, Papademetriou V, Tsiachris D, Kasiakogias A, Kordalis A, Thomopoulos C, et al. Impact of multielectrode renal sympathetic denervation on short-term blood pressure variability in patients with drug-resistant hypertension. Insights from EnligHTN I study. Int J Cardiol 2015; 180:237-242.

9. Ewen S, Dörr O, Ukena C, Linz D, Cremers B, Laufs U, et al. Blood pressure variability after catheter-based renal sympathetic denervation in patients with resistant hypertension. J Hypertens 2015; 33:25122518.

10. Bhatt DL, Kandzari DE, O'Neill WW, D'Agostino R, Flack JM, Katzen BT, SYMPLICITY HTN-3 Investigators. A controlled trial of renal denervation for resistant hypertension. N Engl J Med 2014; 370: 1393-1401. 
11. Fadl Elmula FE, Jin Y, Yang WY, Thijs L, Lu YC, Larstorp AC, et al., European Network Coordinating Research On Renal Denervation (ENCOReD) Consortium. Meta-analysis of randomized controlled trials of renal denervation in treatment-resistant hypertension. Blood Press 2015; 24:263-274.

12. Mahfoud F, Böhm M, Azizi M, Pathak A, Durand Zaleski I, Ewen S, et al. Proceedings from the European clinical consensus conference for renal denervation: considerations on future clinical trial design. Eur Heart J 2015; 36:2219-2227.

13. White WB, Galis ZS, Henegar J, Kandzari DE, Victor R, Sica D, et al. Renal denervation therapy for hypertension: pathways for moving development forward. J Am Soc Hypertens 2015; 9:341-350.

14. Persu A, Jin Y, Azizi M, Baelen M, Völz S, Elvan A, et al., European Network COordinating research on Renal Denervation (ENCOReD). Blood pressure changes after renal denervation at 10 European expert centers. J Hum Hypertens 2014; 28:150-156.

15. Schmieder RE, Redon J, Grassi G, Kjeldsen JE, Mancia G, Narkiewicz K, et al. ESH position paper: renal denervation - an interventional therapy of resistant hypertension. J Hypertens 2012; 30:837-841.

16. O'Brien E, Parati G, Stergiou G, Asmar R, Beilin L, Bilo G, et al. European society of hypertension position paper on ambulatory blood pressure monitoring. J Hypertens 2013; 31:1731-1768.

17. Staessen JA, Bieniaszewski L, O'Brien ET, Imai Y, Fagard R. An epidemiological approach to ambulatory blood pressure monitoring: the Belgian population study. Blood Press Monit 1996; 1:13-26.

18. Fagard R, Brguljan J, Thijs L, Staessen J. Prediction of the actual awake and asleep blood pressures by various methods of $24 \mathrm{~h}$ pressure analysis. J Hypertens 1996; 14:557-563.

19. Pringle E, Phillips C, Thijs L, Davidson C, Staessen JA, de Leeuw PW, et al., Syst-Eur investigators. Systolic blood pressure variability as a risk factor for stroke and cardiovascular mortality in the elderly hypertensive population. J Hypertens 2003; 21:2251-2257.

20. Hansen TW, Thijs L, Li Y, Boggia J, Kikuya M, Björklund-Bodegård K, et al., International Database on Ambulatory Blood Pressure in Relation to Cardiovascular Outcomes Investigators. Prognostic value of readingto-reading blood pressure variability over $24 \mathrm{~h}$ in 8938 subjects from 11 populations. Hypertension 2010; 55:1049-1057.

21. Mena L, Pintos S, Queipo NV, Aizpurua JA, Maestre G, Sulbaran T. A reliable index for the prognostic significance of blood pressure variability. J Hypertens 2005; 23:505-511.

22. Bilo G, Giglio A, Styczkiewicz K, Caldara G, Maronati A, KaweckaJaszcz K, et al. A new method for assessing 24-h blood pressure variability after excluding the contribution of nocturnal blood pressure fall. J Hypertens 2007; 25:2058-2066.

23. Rothwell PM, Howard SC, Dolan E, O'Brien E, Dobson JE, Dahlöf B, et al. Prognostic significance of visit-to-visit variability, maximum systolic blood pressure, and episodic hypertension. Lancet 2010; 375:895-905.

24. Levey AS, Stevens LA, Schmid CH, Zhang YL, Castro AF 3rd, Feldman HI, et al., CKD-EPI (Chronic Kidney Disease Epidemiology Collaboration). A new equation to estimate glomerular filtration rate. Ann Intern Med 2009; 150:604-612.

25. Parati G, Stergiou G, O'Brien E, Asmar R, Beilin L, Bilo G, et al., European Society of Hypertension Working Group on Blood Pressure Monitoring and Cardiovascular Variability. European Society of Hypertension practice guidelines for ambulatory blood pressure monitoring. J Hypertens 2014; 32:1359-1366.

26. Asayama K, Wei F, Hara A, Hansen TW, Li Y, Staessen JA. Prognosis in relation to blood pressure variability. Hypertension 2015; 65: $1170-1179$.

\section{Reviewers' Summary Evaluations}

\section{Reviewer 1}

Studies assessing the effects of renal denervation (RDN) on sympathetic nervous system (SNS) activity in patients with treatment-resistant hypertension have shown contradictory results. The present meta-analysis including patients of the ENCOReD network showed that RDN was associated with a decrease in BP variability independent of the BP level. The
27. Hara A, Thijs L, Asayama K, Jacobs L, Wang J, Staessen JA. Randomised double-blind comparison of placebo and active drugs for effects on risks associated with blood pressure variability in the systolic hypertension in Europe trial. PLoS One 2014; 9:1-18.

28. Ukena C, Mahfoud F, Spies A, Kindermann I, Linz D, Cremers B, et al Effects of renal sympathetic denervation on heart rate and atrioventricular conduction in patients with resistant hypertension. Int J Cardiol 2013; 167:2846-2851.

29. Böhm M, Ukena C, Ewen S, Linz D, Zivanovic I, Hoppe U, et al., Global SYMPLICITY Registry Investigators. Renal denervation reduces office and ambulatory heart rate in patients with uncontrolled hypertension: 12-month outcomes from the global SYMPLICITY registry. J Hypertens 2016; 34:2480-2486.

30. Schutte R, Thijs L, Liu YP, Asayama K, Jin Y, Odili A, et al. Withinsubject blood pressure level-not variability-predicts fatal and nonfatal outcomes in a general population. Hypertension 2012; 60:1138-1147.

31. Protogerou AD, Safar ME, Iaria P, Safar H, Le Dudal K, Filipovsky J, et al. Diastolic blood pressure and mortality in the elderly with cardiovascular disease. Hypertension 2007; 50:172-180.

32. Caliva FS, Harris JF, Lyons RH. Peripheral resistance in hypertension following the abolition of local sympathetic tone. Circulation 1959; 19:564-569.

33. Ewen S, Cremers B, Meyer MR, Donazzan L, Kindermann I, Ukena C, et al. Blood pressure changes after catheter-based renal denervation are related to reductions in total peripheral resistance. J Hypertens 2015; 33:2519-2525.

34. Miura Y, Kobayashi K, Sakuma H, Tomioka H, Adachi M, Yoshinaga K. Plasma noradrenaline concentrations and haemodynamics in the early stage of essential hypertension. Clin Sci Mol Med Suppl 1978; 4:69s-71s.

35. Mulvany MJ. Are vascular abnormalities a primary cause or secondary consequence of hypertension? Hypertension 1991; 18 (3 Suppl): I52-57.

36. de Simone G, Pasanisi F. Systolic, diastolic and pulse pressure: pathophysiology. Ital Heart J Suppl 2001; 2:359-362.

37. Ewen S, Ukena C, Linz D, Kindermann I, Cremers B, Laufs U, et al. Reduced effect of percutaneous renal denervation on blood pressure in patients with isolated systolic hypertension. Hypertension 2015; 65:193-199.

38. de la Sierra A, Pareja J, Armario P, Barrera Á, Yun S, Vázquez S, et al. Renal denervation vs. spironolactone in resistant hypertension: effects on circadian patterns and blood pressure variability. Am J Hypertens 2017; 30:37-41.

39. Grassi G, Seravalle G, Brambilla G, Trabattoni D, Cuspidi C, Corso R, et al. Blood pressure responses to renal denervation precede and are independent of the sympathetic and baroreflex effects. Hypertension 2015; 65:1209-1216.

40. Fadl Elmula FE, Larstorp AC, Kjeldsen SE, Persu A, Jin Y, Staessen JA. Renal sympathetic denervation after Symplicity HTN-3 and therapeutic drug monitoring in severe hypertension. Front Physiol 2015; 6:9.

41. Gal P, de Jong MR, Smit JJ, Adiyaman A, Staessen JA, Elvan A. Blood pressure response to renal nerve stimulation in patients undergoing renal denervation: a feasibility study. J Hum Hypertens 2015; 29:292295.

42. Azizi M, Sapoval M, Gosse P, Monge M, Bobrie G, Delsart P, et al. Renal Denervation for Hypertension (DENERHTN) investigators. Optimum and stepped care standardised antihypertensive treatment with or without renal denervation for resistant hypertension (DENERHTN): a multicentre, open-label, randomised controlled trial. Lancet 2015; 385:1957-1965.

weakness inherent to all published studies assessing the effects of RDN so far is the lack of hard-points results (i.e. $\mathrm{CV}$ morbidity and mortality). This point is also true for SNS activity and its role as an important modifiable determinant of CV morbidity and mortality in hypertensive patients.

\section{Reviewer 2}

Persu et al. performed patient-level meta-analysis of several cohorts of patients who underwent renal denervation 
(RDN). The authors demonstrate: 1) a modest but significant decrease in 24-h blood pressure variability (BPV) six months after the procedure; 2) a weak but significant correlation between baseline BPV and blood pressure response to RDN. The study has the strength of relatively large sample size and has considered several BPV indices.
Moreover, it takes into account the confounding effect of the relationship between BPV and blood pressure level. Given the modest strength of the observed relationships, direct clinical applicability of the findings is limited. Nonetheless, the results may stimulate further research on haemodynamic predictors of the response to RDN. 